\title{
Jindalrae and Gaenari numbers and polynomials in connection with Jindalrae-Stirling numbers
}

\author{
Taekyun Kim ${ }^{1,2}$, Dae San Kim ${ }^{3}$ Lee-Chae Jang ${ }^{4 *}$ and Hyunseok Lee ${ }^{2}$
}

\section{"Correspondence:}

lcjang@konkuk.ac.kr

${ }^{4}$ Graduate School of Education,

Konkuk University, Seoul, Republic

of Korea

Full list of author information is

available at the end of the article

\begin{abstract}
The aim of this paper is to study Jindalrae and Gaenari numbers and polynomials in connection with Jindalrae-Stirling numbers of the first and second kinds. For this purpose, we first introduce Jindalrae-Stirling numbers of the first and second kinds as extensions of the notions of the degenerate Stirling numbers of the first and second kinds, and deduce several relations involving those special numbers. Then we introduce Jindalrae and Gaenari numbers and polynomials and obtain some explicit expressions and identities associated with those numbers and polynomials. In addition, we interpret our results by using umbral calculus.
\end{abstract}

MSC: 11B73; 11B83;05A19

Keywords: Jindalrae numbers and polynomials; Gaenari numbers and polynomials; Degenerate Stirling numbers of the first kind; Degenerate Stirling numbers of the second kind

\section{Introduction and preliminaries}

Let $n$ be a nonnegative integer. Then the Stirling numbers of the first kind are defined as

$$
(x)_{n}=\sum_{l=0}^{n} S_{1}(n, l) x^{l} \quad(\text { see }[5])
$$

where $(x)_{0}=1,(x)_{n}=x(x-1) \cdots(x-n+1)(n \geq 1)$, whereas the Stirling numbers of the second kind are given by

$$
x^{n}=\sum_{l=0}^{n} S_{2}(n, l)(x)_{l} \quad(\text { see }[10,18])
$$

By (1) and (2), we get

$$
\frac{1}{k !}\left(e^{t}-1\right)^{k}=\sum_{n=k}^{\infty} S_{2}(n, k) \frac{t^{n}}{n !} \quad(k \geq 0)
$$

(c) The Author(s) 2020. This article is licensed under a Creative Commons Attribution 4.0 International License, which permits use sharing, adaptation, distribution and reproduction in any medium or format, as long as you give appropriate credit to the original author(s) and the source, provide a link to the Creative Commons licence, and indicate if changes were made. The images or other third party material in this article are included in the article's Creative Commons licence, unless indicated otherwise in a credit line to the material. If material is not included in the article's Creative Commons licence and your intended use is not permitted by statutory regulation or exceeds the permitted use, you will need to obtain permission directly from the copyright holder. To view a copy of this licence, visit http://creativecommons.org/licenses/by/4.0/. 
and

$$
\frac{1}{k !}(\log (1+t))^{k}=\sum_{n=k}^{\infty} S_{1}(n, k) \frac{t^{n}}{n !} \quad(k \geq 0) .
$$

It is well known that the Bell polynomials are defined as

$$
e^{x\left(e^{t}-1\right)}=\sum_{n=0}^{\infty} B_{n}(x) \frac{t^{n}}{n !} \quad(\text { see }[13,19]) .
$$

When $x=1, B_{n}=B_{n}(1)$ are called the Bell numbers.

For $0 \neq \lambda \in \mathbb{R}$, the degenerate exponential functions are defined by

$$
e_{\lambda}^{x}(t)=\sum_{n=0}^{\infty} \frac{(x)_{n, \lambda}}{n !} t^{n}, \quad e_{\lambda}(t)=e_{\lambda}^{1}(t)=\sum_{n=0}^{\infty} \frac{(1)_{n, \lambda}}{n !} t^{n} \quad(\text { see }[12,21]),
$$

where $(x)_{0, \lambda}=1,(x)_{n, \lambda}=x(x-\lambda)(x-2 \lambda) \cdots(x-(n-1) \lambda)(n \geq 1)$.

Let $\log _{\lambda}(t)$ be the compositional inverse of $e_{\lambda}(t)$, called the degenerate logarithm function, such that $\log _{\lambda}\left(e_{\lambda}(t)\right)=e_{\lambda}\left(\log _{\lambda} t\right)=t$.

Then we note that

$$
\log _{\lambda}(1+t)=\frac{1}{\lambda}\left((1+t)^{\lambda}-1\right)=\sum_{n=1}^{\infty} \lambda^{n-1}(1)_{n, \frac{1}{\lambda}} \frac{t^{n}}{n !} \quad(\text { see }[14]) .
$$

By (7), we get $\lim _{\lambda \rightarrow 0} \log _{\lambda}(1+t)=\log (1+t)$.

In [14], the degenerate Stirling numbers of the first kind are defined by

$$
(x)_{n}=\sum_{l=0}^{n} S_{1, \lambda}(n, l)(x)_{l, \lambda} .
$$

As an inversion formula of (8), the degenerate Stirling numbers of the second kind are defined by

$$
(x)_{n, \lambda}=\sum_{k=0}^{n} S_{2, \lambda}(n, k)(x)_{k} \quad(n \geq 0)(\text { see }[16]) .
$$

By (8) and (9), we get

$$
\frac{1}{k !}\left(\log _{\lambda}(1+t)\right)^{k}=\sum_{n=k}^{\infty} S_{1, \lambda}(n, k) \frac{t^{n}}{n !} \quad(k \geq 0)(\text { see [14] })
$$

and

$$
\frac{1}{k !}\left(e_{\lambda}(t)-1\right)^{k}=\sum_{n=k}^{\infty} S_{2, \lambda}(n, k) \frac{t^{n}}{n !} \quad(k \geq 0)(\text { see }[16]) .
$$

We define the degenerate Bell polynomials $B_{n, \lambda}(x)$ by

$$
e_{\lambda}^{x}\left(e_{\lambda}(t)-1\right)=\sum_{n=0}^{\infty} B_{n, \lambda}(x) \frac{t^{n}}{n !} .
$$


When $x=1, B_{n, \lambda}=B_{n, \lambda}(1)$ are called the degenerate Bell numbers.

From (6) and (10), we note that

$$
\begin{aligned}
e_{\lambda}^{x}\left(e_{\lambda}(t)-1\right) & =\sum_{k=0}^{\infty}(x)_{k, \lambda} \frac{1}{k !}\left(e_{\lambda}(t)-1\right)^{k} \\
& =\sum_{k=0}^{\infty}(x)_{k, \lambda} \sum_{n=k}^{\infty} S_{2, \lambda}(n, k) \frac{t^{n}}{n !} \\
& =\sum_{n=0}^{\infty}\left(\sum_{k=0}^{n}(x)_{k, \lambda} S_{2, \lambda}(n, k)\right) \frac{t^{n}}{n !} .
\end{aligned}
$$

By (12) and (13), we get

$$
B_{n, \lambda}(x)=\sum_{k=0}^{n} S_{2, \lambda}(n, k)(x)_{k, \lambda} \quad(n \geq 0) .
$$

Here we note that the so-called new type degenerate Bell polynomials $\operatorname{Bel}_{n, \lambda}(x)$, which are different from the degenerate Bell polynomials just introduced, have been considered recently in [19]. They are defined by the generating function $e_{\lambda}^{x}\left(e^{t}-1\right)=\sum_{n=0}^{\infty} \operatorname{Bel}_{n, \lambda}(x) \frac{t^{n}}{n !}$, so that $\operatorname{Bel}_{n, \lambda}(x)=\sum_{k=0}^{n} S_{2}(n, k)(x)_{k, \lambda}(n \geq 0)$.

In $[2,3]$, Carlitz initiated a study of the degenerate Bernoulli and Euler polynomials and numbers, which are degenerate versions of the usual Bernoulli and Euler polynomials and numbers (see $[1,22,25,30,31])$. In recent years, studying degenerate versions of quite a few special polynomials and numbers has regained lively interest of some mathematicians and yielded many interesting results (see [4-7, 10, 12-17, 19-21, 23, 24, 26-28]). Here we note that such degenerate versions of many special polynomials and numbers have been investigated by employing different tools like generating functions, combinatorial methods, umbral calculus techniques, probability theory, $p$-adic analysis, differential equations, etc.

The aim of the present paper is to study Jindalrae and Gaenari polynomials and numbers in connection with Jindalrae-Stirling numbers of the first and second kinds, and find some arithmetic and combinatorial results on those polynomials and numbers. First, we define Jindalrae-Stirling numbers of the first and second kinds as extensions of the notions of the degenerate Stirling numbers of the first and second kinds, and find some relations involving those special numbers. Then we introduce Jindalrae and Gaenari numbers and polynomials and obtain some explicit expressions and identities associated with those numbers and polynomials. In addition, we interpret our results by using umbral calculus.

Further continuation of the present work would be to find some applications of our results in science and engineering as well as in mathematics. We outlined possible applications of our results to other areas of mathematics, namely to probability, differential equations, and identities of symmetry, in the last section. However, some applications to areas other than mathematics will require considerable amount of work. We hope that we will be able to find such applications in the near future.

This paper is organized as follows. In Sect. 1, we go over some necessary stuff that is needed throughout this paper. This includes the degenerate exponential functions, the degenerate logarithm function, the degenerate Stirling numbers of the first and second 
kinds, and the degenerate Bell numbers. Here we note that the degenerate Bell polynomials $B_{n, \lambda}(x)$ (see (12)) are different from the partially degenerate Bell polynomials bel ${ }_{n, \lambda}(x)$ in [15] and also from the new type degenerate Bell polynomials $\operatorname{Bel}_{n, \lambda}(x)$ in [19]. In Sect. 2, we introduce Jindalrae-Stirling numbers of the first and second kinds, as extensions of the notions of the degenerate Stirling numbers of the first and second kinds and find some relations connecting those special numbers, the degenerate Stirling numbers of the first and second kinds and the degenerate Bell numbers and polynomials. Then we introduce Jindalrae numbers and polynomials, as an extension of the notion of the degenerate Bell numbers and polynomials, and Gaenari numbers and polynomials and find some explicit expressions and identities involving those numbers and polynomials, Jindalrae-Stirling numbers of the first and second kinds, the degenerate Stirling numbers of the first and second kinds, and the degenerate Bell polynomials. In Sect. 3, we interpret the results in Sect. 2 by means of umbral calculus. Finally, we conclude this paper in Sect. 4 .

\section{Jindalrae and Gaenari numbers and polynomials}

By replacing $t$ by $e^{t}-1$ in (3), we get

$$
\begin{aligned}
\frac{1}{k !}\left(e^{e^{t}-1}-1\right)^{k} & =\sum_{m=k}^{\infty} S_{2}(m, k) \frac{1}{m !}\left(e^{t}-1\right)^{m} \\
& =\sum_{m=k}^{\infty} S_{2}(m, k) \sum_{n=m}^{\infty} S_{2}(n, m) \frac{t^{n}}{n !} \\
& =\sum_{n=k}^{\infty}\left(\sum_{m=k}^{n} S_{2}(m, k) S_{2}(n, m)\right) \frac{t^{n}}{n !} .
\end{aligned}
$$

Let

$$
\frac{1}{k !}\left(e^{e^{t}-1}-1\right)^{k}=\sum_{n=k}^{\infty} T(n, k) \frac{t^{n}}{n !} .
$$

Then, by (15) and (16), we get

$$
T(n, k)=\sum_{m=k}^{\infty} S_{2}(n, m) S_{2}(m, k),
$$

where $n, k \geq 0$, with $n \geq k$.

Further, we have

$$
\begin{aligned}
\frac{1}{k !}\left(e^{e^{t}-1}-1\right)^{k} & =\frac{1}{k !}\left(\sum_{n=1}^{\infty} B_{n} \frac{t^{n}}{n !}\right)^{k} \\
& =\frac{1}{k !} \sum_{n=k}^{\infty}\left(\sum_{n_{1}+\cdots+n_{k}=n}\left(\begin{array}{c}
n \\
n_{1}, \ldots, n_{k}
\end{array}\right) B_{n_{1}} \cdots B_{n_{k}}\right) \frac{t^{n}}{n !},
\end{aligned}
$$


where the inner sum runs over all positive integers $n_{1}, \ldots, n_{k}$, with $n_{1}+\cdots+n_{k}=n$. Thus, by (16), (17), and (18), we get

$$
\begin{aligned}
T(n, k) & =\frac{1}{k !} \sum_{n_{1}+\cdots+n_{k}=n}\left(\begin{array}{c}
n \\
n_{1}, \ldots, n_{k}
\end{array}\right) B_{n_{1}} \cdots B_{n_{k}} \\
& =\sum_{m=k}^{n} S_{2}(n, m) S_{2}(m, k) .
\end{aligned}
$$

Note that $T(n, 1)=\sum_{m=1}^{n} S_{2}(n, m)=B_{n}(n \geq 1)$.

For $k \geq 0$, as an extension of the notion of the degenerate Stirling numbers of the second kind, we define Jindalrae-Stirling numbers of the second kind (see (9) and (11)) by

$$
\frac{1}{k !}\left(e_{\lambda}\left(e_{\lambda}(t)-1\right)-1\right)^{k}=\sum_{n=k}^{\infty} S_{J, \lambda}^{(2)}(n, k) \frac{t^{n}}{n !} .
$$

On the other hand,

$$
\begin{aligned}
\frac{1}{k !}\left(e_{\lambda}\left(e_{\lambda}(t)-1\right)-1\right)^{k} & =\sum_{m=k}^{\infty} S_{2, \lambda}(m, k) \sum_{n=m}^{\infty} S_{2, \lambda}(n, m) \frac{t^{n}}{n !} \\
& =\sum_{n=k}^{\infty}\left(\sum_{m=k}^{n} S_{2, \lambda}(m, k) S_{2, \lambda}(n, m)\right) \frac{t^{n}}{n !} .
\end{aligned}
$$

Therefore, by (20) and (21), we obtain the following theorem.

Theorem 1 For $n, k \geq 0$, with $n \geq k$, we have

$$
S_{J, \lambda}^{(2)}(n, k)=\sum_{m=k}^{n} S_{2, \lambda}(n, m) S_{2, \lambda}(m, k) .
$$

When $k=1$, we have

$$
\begin{aligned}
\sum_{n=1}^{\infty} S_{J, 2}^{(2)}(n, 1) \frac{t^{n}}{n !} & =e_{\lambda}\left(e_{\lambda}(t)-1\right)-1 \\
& =\sum_{n=0}^{\infty} B_{n, \lambda} \frac{t^{n}}{n !}-1=\sum_{n=1}^{\infty} B_{n, \lambda} \frac{t^{n}}{n !} .
\end{aligned}
$$

For $n \geq 1$, we have

$$
\begin{aligned}
S_{J, \lambda}^{(2)}(n, 1) & =B_{n, \lambda} \\
& =\sum_{m=1}^{n} S_{2, \lambda}(n, m) S_{2, \lambda}(m, 1) \\
& =\sum_{m=1}^{n} S_{2, \lambda}(n, m)(1)_{m, \lambda} .
\end{aligned}
$$


For $k \geq 0$, by replacing $t$ by $\log _{\lambda}(1+t)$ in (20), we get

$$
\begin{aligned}
\frac{1}{k !}\left(e_{\lambda}(t)-1\right)^{k} & =\sum_{m=k}^{\infty} S_{J, \lambda}^{(2)}(m, k) \frac{1}{m !}\left(\log _{\lambda}(1+t)\right)^{m} \\
& =\sum_{m=k}^{\infty} S_{J, \lambda}^{(2)}(m, k) \sum_{n=m}^{\infty} S_{1, \lambda}(n, m) \frac{t^{n}}{n !} \\
& =\sum_{n=k}^{\infty}\left(\sum_{m=k}^{n} S_{J, \lambda}^{(2)}(m, k) S_{1, \lambda}(n, m)\right) \frac{t^{n}}{n !} .
\end{aligned}
$$

Therefore, by (10) and (23), we obtain the following theorem.

Theorem 2 For $n, k \geq 0$, with $n \geq k$, we have

$$
S_{2, \lambda}(n, k)=\sum_{m=k}^{n} S_{J, \lambda}^{(2)}(m, k) S_{1, \lambda}(n, m)
$$

When $k=1$, we have

$$
S_{2, \lambda}(n, 1)=\sum_{m=1}^{n} S_{J, \lambda}^{(2)}(m, 1) S_{1, \lambda}(n, m)=\sum_{m=1}^{n} B_{m, \lambda} S_{1, \lambda}(n, m) .
$$

It is easy to show that $S_{2, \lambda}(n, 1)=(1)_{n, \lambda}(n \geq 1)$. Therefore, by (24), we obtain the following corollary.

Corollary 3 For $n \in \mathbb{N}$, we have

$$
\sum_{m=1}^{n} B_{m, \lambda} S_{1, \lambda}(n, m)=(1)_{n, \lambda}
$$

As an inversion formula of (20) and an extension of the notion of the degenerate Stirling numbers of the first kind (see (8) and (10)), we define Jindalrae-Stirling numbers of the first kind by

$$
\frac{1}{k !}\left(\log _{\lambda}\left(\log _{\lambda}(1+t)+1\right)\right)^{k}=\sum_{n=k}^{\infty} S_{J, \lambda}^{(1)}(n, k) \frac{t^{n}}{n !} \quad(k \geq 0) .
$$

We note that

$$
\begin{aligned}
\frac{1}{k !}\left(\log _{\lambda}\left(\log _{\lambda}(1+t)+1\right)\right)^{k} & =\sum_{m=k}^{\infty} S_{1, \lambda}(m, k) \frac{1}{m !}\left(\log _{\lambda}(1+t)\right)^{m} \\
& =\sum_{m=k}^{\infty} S_{1, \lambda}(m, k) \sum_{n=m}^{\infty} S_{1, \lambda}(n, m) \frac{t^{n}}{n !} \\
& =\sum_{n=k}^{\infty}\left(\sum_{m=k}^{n} S_{1, \lambda}(m, k) S_{1, \lambda}(n, m)\right) \frac{t^{n}}{n !} .
\end{aligned}
$$

Therefore, by (25) and (26), we obtain the following theorem. 
Theorem 4 For $n, k \geq 0$, with $n \geq k$, we have

$$
S_{J, \lambda}^{(1)}(n, k)=\sum_{m=k}^{n} S_{1, \lambda}(n, m) S_{1, \lambda}(m, k) .
$$

When $k=1$, we have

$$
\begin{aligned}
S_{J, \lambda}^{(1)}(n, 1) & =\sum_{m=1}^{n} S_{1, \lambda}(m, 1) S_{1, \lambda}(n, m) \\
& =\sum_{m=1}^{n}(m-1) !\left(\begin{array}{c}
\lambda-1 \\
m-1
\end{array}\right) S_{1, \lambda}(n, m) .
\end{aligned}
$$

Corollary 5 For $n \geq 1$, we have

$$
S_{J, \lambda}^{(1)}(n, 1)=\sum_{m=1}^{n}(m-1) !\left(\begin{array}{c}
\lambda-1 \\
m-1
\end{array}\right) S_{1, \lambda}(n, m) .
$$

From (20), we note that

$$
\begin{aligned}
\sum_{n=k}^{\infty} S_{J, \lambda}^{(2)}(n, k) \frac{t^{n}}{n !} & =\frac{1}{k !}\left(e_{\lambda}\left(e_{\lambda}(t)-1\right)-1\right)^{k} \\
& =\frac{1}{k !} \sum_{l=0}^{k}\left(\begin{array}{l}
k \\
l
\end{array}\right)(-1)^{k-l} e_{\lambda}^{l}\left(e_{\lambda}(t)-1\right) \\
& =\sum_{n=0}^{\infty}\left(\frac{1}{k !} \sum_{l=0}^{k}\left(\begin{array}{l}
k \\
l
\end{array}\right)(-1)^{k-l} B_{n, \lambda}(l)\right) \frac{t^{n}}{n !},
\end{aligned}
$$

where $k$ is a nonnegative integer.

By comparing the coefficients on both sides of (28), we get

$$
\frac{1}{k !} \sum_{l=0}^{k}\left(\begin{array}{l}
k \\
l
\end{array}\right)(-1)^{k-l} B_{n, \lambda}(l)= \begin{cases}S_{J, \lambda}^{(2)}(n, k), & \text { if } n \geq k \\
0, & \text { if } 0 \leq n<k\end{cases}
$$

Therefore, by (29), we obtain the following theorem.

Theorem 6 For $n, k \geq 0$, with $n \geq k$, we have

$$
S_{J, \lambda}^{(2)}(n, k)=\frac{1}{k !} \sum_{l=0}^{k}\left(\begin{array}{l}
k \\
l
\end{array}\right)(-1)^{k-l} B_{n, \lambda}(l) .
$$

Now, we observe that

$$
\begin{aligned}
\sum_{k=0}^{\infty}(x)_{k, \lambda} \frac{1}{k !}\left(\log _{\lambda}\left(\log _{\lambda}(1+t)+1\right)\right)^{k} & =\sum_{k=0}^{\infty}(x)_{k, \lambda} \sum_{n=k}^{\infty} S_{J, \lambda}^{(1)}(n, k) \frac{t^{n}}{n !} \\
& =\sum_{n=0}^{\infty}\left(\sum_{k=0}^{n}(x)_{k, \lambda} S_{J, \lambda}^{(1)}(n, k)\right) \frac{t^{n}}{n !} .
\end{aligned}
$$


On the other hand,

$$
\begin{aligned}
\sum_{k=0}^{\infty}(x)_{k, \lambda} \frac{1}{k !}\left(\log _{\lambda}\left(\log _{\lambda}(1+t)+1\right)\right)^{k} & =e_{\lambda}^{x}\left(\log _{\lambda}\left(\log _{\lambda}(1+t)+1\right)\right) . \\
& =\left(\log _{\lambda}(1+t)+1\right)^{x} \\
& =\sum_{l=0}^{\infty}(x)_{l} \frac{1}{l !}\left(\log _{\lambda}(1+t)\right)^{l} \\
& =\sum_{l=0}^{\infty}(x)_{l} \sum_{n=l}^{\infty} S_{1, \lambda}(n, l) \frac{t^{n}}{n !} \\
& =\sum_{n=0}^{\infty}\left(\sum_{l=0}^{n} S_{1, \lambda}(n, l)(x)_{l}\right) \frac{t^{n}}{n !} .
\end{aligned}
$$

Thus, by (30) and (31), we get

$$
\sum_{l=0}^{n} S_{1, \lambda}(n, l)(x)_{l}=\sum_{k=0}^{n} S_{J, \lambda}^{(1)}(n, k)(x)_{k, \lambda} \quad(n \geq 0) .
$$

From (9) and (32), we can derive the following Eq. (33):

$$
\begin{aligned}
\sum_{l=0}^{n} S_{1, \lambda}(n, l)(x)_{l} & =\sum_{k=0}^{n} S_{J, \lambda}^{(1)}(n, k)(x)_{k, \lambda} \\
& =\sum_{k=0}^{n} S_{J, \lambda}^{(1)}(n, k) \sum_{l=0}^{k} S_{2, \lambda}(k, l)(x)_{l} \\
& =\sum_{l=0}^{n}\left(\sum_{k=l}^{n} S_{J, \lambda}^{(1)}(n, k) S_{2, \lambda}(k, l)\right)(x)_{l} .
\end{aligned}
$$

Therefore, by comparing the coefficients as both sides of (33), we obtain the following theorem.

Theorem 7 For $n, l \geq 0$, we have

$$
S_{1, \lambda}(n, l)=\sum_{k=l}^{n} S_{J, \lambda}^{(1)}(n, k) S_{2, \lambda}(k, l)
$$

When $l=1$, we have

$$
\begin{aligned}
S_{1, \lambda}(n, 1) & =\sum_{k=1}^{n} S_{J, \lambda}^{(1)}(n, k) S_{2, \lambda}(k, 1) \\
& =\sum_{k=1}^{n}(1)_{k, \lambda} S_{J, \lambda}^{(1)}(n, k) .
\end{aligned}
$$


As an extension of the degenerate Bell polynomials in (12), the Jindalrae polynomials are defined by

$$
e_{\lambda}^{x}\left(e_{\lambda}\left(e_{\lambda}(t)-1\right)-1\right)=\sum_{n=0}^{\infty} J_{n, \lambda}(x) \frac{t^{n}}{n !} .
$$

When $x=1, J_{n, \lambda}=J_{n, \lambda}(1)$ are called Jindalrae numbers.

From (35), we note that

$$
\begin{aligned}
e_{\lambda}^{x}\left(e_{\lambda}\left(e_{\lambda}(t)-1\right)-1\right) & =\sum_{k=0}^{\infty}(x)_{k, \lambda} \frac{1}{k !}\left(e_{\lambda}\left(e_{\lambda}(t)-1\right)-1\right)^{k} \\
& =\sum_{k=0}^{\infty}(x)_{k, \lambda} \sum_{n=k}^{\infty} S_{J, \lambda}^{(2)}(n, k) \frac{t^{n}}{n !} \\
& =\sum_{n=0}^{\infty}\left(\sum_{k=0}^{n}(x)_{k, \lambda} S_{J, \lambda}^{(2)}(n, k)\right) \frac{t^{n}}{n !} .
\end{aligned}
$$

Therefore, by (35) and (36), we obtain the following theorem.

Theorem 8 For $n \geq 0$, we have

$$
J_{n, \lambda}(x)=\sum_{k=0}^{n}(x)_{k, \lambda} S_{J, \lambda}^{(2)}(n, k)
$$

In particular, for $x=1$,

$$
J_{n, \lambda}=\sum_{k=0}^{n}(1)_{k, \lambda} S_{J, \lambda}^{(2)}(n, k) .
$$

By replacing $t$ by $\log _{\lambda}(1+t)$ in (35), we get

$$
\begin{aligned}
e_{\lambda}^{x}\left(e_{\lambda}(t)-1\right) & =\sum_{m=0}^{\infty} J_{m, \lambda}(x) \frac{1}{m !}\left(\log _{\lambda}(1+t)\right)^{m} \\
& =\sum_{m=0}^{\infty} J_{m, \lambda}(x) \sum_{n=m}^{\infty} S_{1, \lambda}(n, m) \frac{t^{n}}{n !} \\
& =\sum_{n=0}^{\infty}\left(\sum_{m=0}^{n} J_{m, \lambda}(x) S_{1, \lambda}(n, m)\right) \frac{t^{n}}{n !} .
\end{aligned}
$$

Therefore, by (12) and (37), we obtain the following theorem.

Theorem 9 For $n \geq 0$, we have

$$
B_{n, \lambda}(x)=\sum_{m=0}^{n} J_{m, \lambda}(x) S_{1, \lambda}(n, m) .
$$


In particular,

$$
B_{n, \lambda}=\sum_{m=0}^{n} J_{m, \lambda} S_{1, \lambda}(n, m)
$$

From (20), we note that

$$
\begin{aligned}
e_{\lambda}^{x}\left(e_{\lambda}\left(e_{\lambda}(t)-1\right)-1\right) & =\sum_{m=0}^{\infty} B_{m, \lambda}(x) \frac{1}{m !}\left(e_{\lambda}(t)-1\right)^{m} \\
& =\sum_{m=0}^{\infty} B_{m, \lambda}(x) \sum_{n=m}^{\infty} S_{2, \lambda}(n, m) \frac{t^{n}}{n !} \\
& =\sum_{n=0}^{\infty}\left(\sum_{m=0}^{n} B_{m, \lambda}(x) S_{2, \lambda}(n, m)\right) \frac{t^{n}}{n !} .
\end{aligned}
$$

Therefore, by (35) and (38), we obtain the following theorem.

Theorem 10 For $n \geq 0$, we have

$$
J_{n, \lambda}(x)=\sum_{m=0}^{n} B_{m, \lambda}(x) S_{2, \lambda}(n, m)
$$

In particular,

$$
J_{n, \lambda}=\sum_{m=0}^{n} B_{m, \lambda} S_{2, \lambda}(n, m)
$$

It is not difficult to show that $\log _{\lambda}\left(\log _{\lambda}(1+t)+1\right)$ is the compositional inverse of $e_{\lambda}\left(e_{\lambda}(t)-1\right)-1$. Now, we consider the Gaenari polynomials given by

$$
e_{\lambda}^{x}\left(\log _{\lambda}\left(\log _{\lambda}(1+t)+1\right)\right)=\sum_{n=0}^{\infty} G_{n, \lambda}(x) \frac{t^{n}}{n !}
$$

When $x=1, G_{n, \lambda}(1)=G_{n, \lambda}$ are called the Gaenari numbers.

From (6), we note that

$$
\begin{aligned}
e_{\lambda}^{x}\left(\log _{\lambda}\left(\log _{\lambda}(1+t)+1\right)\right) & =\sum_{k=0}^{\infty}(x)_{k, \lambda} \frac{1}{k !}\left(\log _{\lambda}\left(\log _{\lambda}(1+t)+1\right)\right)^{k} \\
& =\sum_{k=0}^{\infty}(x)_{k, \lambda} \sum_{n=k}^{\infty} S_{J, \lambda}^{(1)}(n, k) \frac{t^{n}}{n !} \\
& =\sum_{n=0}^{\infty}\left(\sum_{k=0}^{n}(x)_{k, \lambda} S_{J, \lambda}^{(1)}(n, k)\right) \frac{t^{n}}{n !} .
\end{aligned}
$$

Therefore, by (39) and (40), we obtain the following theorem. 
Theorem 11 For $n \geq 0$, we have

$$
G_{n, \lambda}(x)=\sum_{k=0}^{n}(x)_{k, \lambda} S_{J, \lambda}^{(1)}(n, k)
$$

In particular,

$$
G_{n, \lambda}=\sum_{k=0}^{n}(1)_{k, \lambda} S_{J, \lambda}^{(1)}(n, k) .
$$

By replacing $t$ by $e_{\lambda}(t)-1$ in (39), we get

$$
\begin{aligned}
e_{\lambda}^{x}\left(\log _{\lambda}(1+t)\right) & =\sum_{m=0}^{\infty} G_{m, \lambda}(x) \frac{1}{m !}\left(e_{\lambda}(t)-1\right)^{m} \\
& =\sum_{m=0}^{\infty} G_{m, \lambda}(x) \sum_{n=m}^{\infty} S_{2, \lambda}(n, m) \frac{t^{n}}{n !} \\
& =\sum_{n=0}^{\infty}\left(\sum_{m=0}^{n} G_{m, \lambda}(x) S_{2, \lambda}(n, m)\right) \frac{t^{n}}{n !} .
\end{aligned}
$$

On the other hand,

$$
e_{\lambda}^{x}\left(\log _{\lambda}(1+t)\right)=\sum_{n=0}^{\infty}(x)_{n} \frac{t^{n}}{n !}
$$

Therefore, by (41) and (42), we obtain the following theorem.

Theorem 12 For $n \geq 0$, we have

$$
(x)_{n}=\sum_{m=0}^{n} G_{m, \lambda}(x) S_{2, \lambda}(n, m) .
$$

When $x=1$, we have

$$
(1)_{n}=\sum_{m=0}^{n} G_{m, \lambda} S_{2, \lambda}(n, m) \text {. }
$$

By (43), we get

$$
G_{0, \lambda}=1, \quad \sum_{m=0}^{n} G_{m, \lambda} S_{2, \lambda}(n, m)= \begin{cases}1, & \text { if } n=1, \\ 0, & \text { if } n>1 .\end{cases}
$$

From (44), we note that $G_{1, \lambda}=1$. Indeed, we note that

$$
e_{\lambda}^{x}\left(\log _{\lambda}\left(\log _{\lambda}(1+t)+1\right)\right)=(1+\log (1+t))^{x} .
$$


Thus, by (39) and (45), we get

$$
\left(1+\log _{\lambda}(1+t)\right)^{x}=\sum_{n=0}^{\infty} G_{n, \lambda}(x) \frac{t^{n}}{n !}
$$

From (7) and (46), we have

$$
\sum_{n=0}^{\infty} G_{n, \lambda} \frac{t^{n}}{n !}=1+\sum_{n=1}^{\infty} \lambda^{n-1}(1)_{n, 1 / \lambda} \frac{t^{n}}{n !}
$$

Thus, by (47), we get

$$
G_{n, \lambda}=\lambda^{n-1}(1)_{n, 1 / \lambda} \quad(n \geq 1) .
$$

Corollary 13 For $n \geq 1$, we have

$$
G_{n, \lambda}=\lambda^{n-1}(1)_{n, 1 / \lambda} \quad(n \geq 1) .
$$

By replacing $t$ by $e_{\lambda}\left(e_{\lambda}(t)-1\right)-1$ in (39), we get

$$
\begin{aligned}
e_{\lambda}^{x}(t) & =\sum_{m=0}^{\infty} G_{m, \lambda}(x) \frac{1}{m !}\left(e_{\lambda}\left(e_{\lambda}(t)-1\right)^{m}\right. \\
& =\sum_{m=0}^{\infty} G_{m, \lambda}(x) \sum_{n=m}^{\infty} S_{J, \lambda}^{(2)}(n, m) \frac{t^{n}}{n !} \\
& =\sum_{n=0}^{\infty}\left(\sum_{m=0}^{n} G_{m, \lambda}(x) S_{J, \lambda}^{(2)}(n, m)\right) \frac{t^{n}}{n !} .
\end{aligned}
$$

From (6) and (48), we have

$$
(x)_{n, \lambda}=\sum_{m=0}^{n} G_{m, \lambda}(x) S_{J, \lambda}^{(2)}(n, m) \quad(n \geq 0) .
$$

In particular,

$$
(1)_{n, \lambda}=\sum_{m=0}^{n} G_{m, \lambda} S_{J, \lambda}^{(2)}(n, m) \text {. }
$$

From (35), we also note that

$$
\begin{aligned}
e_{\lambda}^{x}(t) & =\sum_{m=0}^{\infty} J_{m, \lambda}(x) \frac{1}{m !}\left(\log _{\lambda}\left(\log _{\lambda}(1+t)+1\right)\right)^{m} \\
& =\sum_{m=0}^{\infty} J_{m, \lambda}(x) \sum_{n=m}^{\infty} S_{J, \lambda}^{(1)}(n, m) \frac{t^{n}}{n !} \\
& =\sum_{n=0}^{\infty}\left(\sum_{m=0}^{n} J_{m, \lambda}(x) S_{J, \lambda}^{(1)}(n, m)\right) \frac{t^{n}}{n !} .
\end{aligned}
$$


Thus, by (6) and (50), we get

$$
(x)_{n, \lambda}=\sum_{m=0}^{n} J_{m, \lambda}(x) S_{J, \lambda}^{(1)}(n, m) \quad(n \geq 0) .
$$

From (49) and (51), we have

$$
\sum_{m=0}^{n} G_{m, \lambda}(x) S_{J, \lambda}^{(2)}(n, m)=\sum_{m=0}^{n} J_{m, \lambda}(x) S_{J, \lambda}^{(1)}(n, m) .
$$

\section{Further remarks}

In this section, we are going to interpret what we obtained in the previous section by means of umbral calculus. First, we will go over some necessary facts about umbral calculus. For more details on umbral calculus, we let the reader refer to [29].

A series $g(t)$ with $O(g(t))=0$ and a series $f(t)$ with $O(f(t))=1$ are respectively called an invertible series and a delta series. Recall here that the order $O(f(t))$ of the nonzero power series $f(t)$ is the smallest integer $k$ for which the coefficient of $t^{k}$ does not vanish. Let $g(t)$ be an invertible series, and let $f(t)$ be a delta series. Then there exists a unique sequence $s_{n}(x)$ of polynomials such that $\left\langle g(t) f(t)^{k} \mid s_{n}(x)\right\rangle=n ! \delta_{n, k}$ for $n, k \geq 0$ (see [29]). The sequence $s_{n}(x)$ is called the Sheffer sequence for the Sheffer pair $(g(t), f(t))$, which is denoted by $s_{n}(x) \sim(g(t), f(t))$. In particular, $s_{n}(x)$ is called the associated sequence to $f(t)$ if $s_{n}(x) \sim(1, f(t))$. Further, $s_{n}(x)$ is called the Appell sequence for $g(t)$ if $s_{n}(x) \sim(g(t), t)$. It is well known that $s_{n}(x) \sim(g(t), f(t))$ if and only if

$$
\frac{1}{g(\bar{f}(t))} e^{x \bar{f}(t)}=\sum_{n \geq 0} s_{n}(x) \frac{t^{n}}{n !}
$$

where $\bar{f}(t)$ is the compositional inverse of $f(t)$ determined by $f(\bar{f}(t))=\bar{f}(f(t))=t$.

For each nonnegative integer $m$, the $m$ th power of an invertible series $g(t)$ will be indicated by $(g(t))^{m}$, while the compositional powers of a delta series $f(t)$ will be denoted by $f^{m}(t)=f \circ f \circ \cdots \circ f(t)$ ( $m$ times). For $p_{n}(x)$ and $q_{n}(x)=\sum_{k=0}^{n} q_{n, k} t^{k}$, the umbral composition of $q_{n}(x)$ with $p_{n}(x)$, denoted by $q_{n} \circ p_{n}(x)$, is defined by $q_{n} \circ p_{n}(x)=\sum_{k=0}^{n} q_{n, k} p_{k}(x)$.

The next result is stated in Theorem 3.5.5 of [29].

Theorem 14 The set of Sheffer sequences forms a group under operation of umbral composition. If $s_{n}(x) \sim(g(t), f(t))$ and $r_{n}(x) \sim(h(t), \ell(t))$, then $r_{n}(x) \circ s_{n}(x) \sim(g(t) h(f(t)), \ell(f(t)))$. The identity under umbral composition is $x^{n} \sim(1, t)$, and the inverse of the sequence $s_{n}(x) \sim(g(t), f(t))$ is the Sheffer sequence for $\left(g(\bar{f}(t))^{-1}, \bar{f}(t)\right)$.

As a corollary, we obtain the following result that will be needed later.

Corollary 15 Let $s_{n}(x) \sim(g(t), f(t))$, and let $r_{n}(x) \sim(1, \ell(t))$. Then, for any positive integer $m$, the generating function for $r_{n}^{(m)} \circ s_{n}(x)$ is obtained from that for $s_{n}(x)$ by substituting $\bar{\ell}^{m}(t)$ for $t$. 
Proof As $r_{n}^{(m)} \circ s_{n}(x) \sim\left(g(t), \ell^{m}(f(t))\right)$, and the compositional inverse of $\ell^{m}(f(t))$ is $\bar{f}\left(\bar{\ell}^{m}(t)\right)$, we have

$$
g\left(\bar{f}\left(\bar{\ell}^{m}(t)\right)\right)^{-1} e^{x \bar{f}\left(\bar{\ell}^{m}(t)\right)}=\sum_{n \geq 0} r_{n}^{(m)} \circ s_{n}(x) \frac{t^{n}}{n !} .
$$

From the definition of umbral composition, we see that the $m$ th power under umbral composition of $r_{n}(x) \sim(h(t), \ell(t))$ is given by

$$
r_{n}^{(m)}(x) \sim\left(\prod_{i=1}^{m-1} h\left(\ell^{i}(t)\right), \ell^{m}(t)\right)
$$

for any positive integer $m$. In particular, this says that, for the Appell sequence $r_{n}(x) \sim$ $(h(t), t)$, we have $r_{n}^{(m)}(x) \sim\left((h(t))^{m}, t\right)$, whereas for the associated sequence $r_{n}(x) \sim(1, \ell(t))$, we have $r_{n}^{(m)}(x) \sim\left(1, \ell^{m}(t)\right)$.

For $n \geq 0$, we write $r_{n}(x)=\sum_{k=0}^{n} r_{n, k} x^{k}=\sum_{k \geq 0} r_{n, k} x^{k}$, where we agree that $r_{i, j}=0$ for all $i<j$. In general, we write

$$
r_{n}^{(m)}(x)=\sum_{k=0}^{n} r_{n, k}^{(m)} x^{k}=\sum_{k \geq 0} r_{n, k}^{(m)} x^{k}
$$

for all $m \in \mathbb{Z}_{>0}$. Then we see that

$$
r_{n, k}^{(m)}=\sum_{\ell_{1}, \ldots, \ell_{m-1}=0}^{n} r_{n, \ell_{1}} r_{\ell_{1}, \ell_{2}} \cdots r_{\ell_{m-1}, k}, \quad m \geq 1,
$$

where we understand $r_{n, k}^{(1)}=r_{n, k}$ for $m=1$.

\subsection{Jindalrae polynomials}

From (35), we note that the Jindalrae polynomials $J_{n, \lambda}(x)$ are given by

$$
\sum_{n=0}^{\infty} J_{n, \lambda}(x) \frac{t^{n}}{n !}=e^{x \bar{f}\left(\bar{\ell}^{2}(t)\right)}
$$

where $f(t)=\frac{1}{\lambda}\left(e^{\lambda t}-1\right), \ell(t)=\log _{\lambda}(1+t)$. Noting that $\bar{f}(t)=\frac{1}{\lambda} \log (1+\lambda t), \bar{\ell}(t)=e_{\lambda}(t)-1$, we have

$$
\begin{aligned}
& e^{x \bar{f}(t)}=e_{\lambda}^{x}(t)=\sum_{n=0}^{\infty}(x)_{n, \lambda} \frac{t^{n}}{n !}, \\
& e^{x \bar{\ell}(t)}=\sum_{n=0}^{\infty} \sum_{k=0}^{n} S_{2, \lambda}(n, k) x^{k} \frac{t^{n}}{n !} .
\end{aligned}
$$

By (58), we let

$$
\begin{aligned}
& s_{n}(x)=(x)_{n, \lambda} \sim\left(1, f(t)=\frac{1}{\lambda}\left(e^{\lambda t}-1\right)\right), \\
& r_{n}(x)=\sum_{k=0}^{n} S_{2, \lambda}(n, k) x^{k} \sim\left(1, \ell(t)=\log _{\lambda}(1+t)\right) .
\end{aligned}
$$


From (54) and (57), we observe that

$$
J_{n, \lambda}(x)=r_{n}^{(2)} \circ s_{n}(x)
$$

As a check, we verify Theorem 8 again by using (60). From (56) and (59), we see that

$$
\begin{aligned}
r_{n}^{(2)}(x) & =\sum_{k=0}^{n} \sum_{l=0}^{n} S_{2, \lambda}(n, l) S_{2, \lambda}(l, k) x^{k} \\
& =\sum_{k=0}^{n} \sum_{l=k}^{n} S_{2, \lambda}(n, l) S_{2, \lambda}(l, k) x^{k} \\
& =\sum_{k=0}^{\infty} S_{J, \lambda}^{(2)}(n, k) x^{k} .
\end{aligned}
$$

Thus, from (59), (60), and (61), we get the desired result as follows:

$$
J_{n, \lambda}(x)=\sum_{k=0}^{n} S_{J, \lambda}^{(2)}(n, k)(x)_{k, \lambda} .
$$

For any positive integer $r$, Korobov polynomials of the first kind of order $r$ are given by

$$
\left(\frac{t}{\log _{\lambda}(1+t)}\right)^{r}(1+t)^{x}=\left(\frac{\lambda t}{(1+t)^{\lambda}-1}\right)^{r}(1+t)^{x}=\sum_{n=0}^{\infty} K_{n,(r)}(x ; \lambda) .
$$

For $x=0, K_{n,(r)}(\lambda)=K_{n,(r)}(0 ; \lambda)$ are called Korobov numbers of the first kind of order $r$. The Korobov polynomials (respectively, numbers) of the first kind are also called the degenerate Bernoulli polynomials (respectively, numbers) of the second kind.

In [9], it was shown that

$$
\begin{aligned}
& \sum_{l_{1}, \ldots, l_{m-1}=0}^{n} S_{2, \lambda}\left(n, l_{1}\right) S_{2, \lambda}\left(l_{1}, l_{2}\right) \cdots S_{2, \lambda}\left(l_{m-1}, k\right) \\
& =\sum_{n \geq l_{1} \geq \cdots \geq l_{m-1} \geq k} S_{2, \lambda}\left(n, l_{1}\right) S_{2, \lambda}\left(l_{1}, l_{2}\right) \cdots S_{2, \lambda}\left(l_{m-1}, k\right) \\
& =\sum_{k_{1}+\cdots+k_{m}=n-k}\left(\begin{array}{c}
n-1 \\
k_{1}, \ldots, k_{m}, k-1
\end{array}\right) \prod_{j=1}^{m} K_{k_{j},\left(n-\sum_{i=j+1}^{m} k_{i}\right)}(\lambda) .
\end{aligned}
$$

Therefore, in the special cases of $m=1$ and $m=2$, we respectively have

$$
\begin{aligned}
& S_{2, \lambda}(n, k)=\left(\begin{array}{l}
n-1 \\
k-1
\end{array}\right) K_{n-k,(n)}(\lambda), \\
& S_{J, \lambda}^{(2)}(n, k)=\sum_{k_{1}+k_{2}=n-k}\left(\begin{array}{c}
n-1 \\
k_{1}, k_{2}, k-1
\end{array}\right) K_{k_{2},(n)}(\lambda) K_{k_{1},\left(n-k_{2}\right)}(\lambda) .
\end{aligned}
$$




\subsection{Gaenari polynomials}

From (39), we recall that the Gaenari polynomials $G_{n, \lambda}(x)$ are given by

$$
\sum_{n=0}^{\infty} G_{n, \lambda}(x) \frac{t^{n}}{n !}=e^{x \bar{f}\left(\vec{\ell}^{2}(t)\right)}
$$

where $f(t)=\frac{1}{\lambda}\left(e^{\lambda t}-1\right), \ell(t)=e_{\lambda}(t)-1$.

Noting that $\bar{f}(t)=\frac{1}{\lambda} \log (1+\lambda t), \bar{\ell}(t)=\log _{\lambda}(1+t)$, we have

$$
\begin{aligned}
e^{x \bar{f}(t)} & =e_{\lambda}^{x}(t)=\sum_{n=0}^{\infty}(x)_{n, \lambda} \frac{t^{n}}{n !}, \\
e^{x \bar{\ell}(t)} & =\sum_{n=0}^{\infty} \sum_{k=0}^{n} S_{1, \lambda}(n, k) x^{k} \frac{t^{n}}{n !} .
\end{aligned}
$$

By (58), we let

$$
\begin{aligned}
& s_{n}(x)=(x)_{n, \lambda} \sim\left(1, f(t)=\frac{1}{\lambda}\left(e^{\lambda t}-1\right)\right), \\
& r_{n}(x)=\sum_{k=0}^{n} S_{1, \lambda}(n, k) x^{k} \sim\left(1, \ell(t)=e_{\lambda}(t)-1\right) .
\end{aligned}
$$

From (54) and (63), we observe that

$$
G_{n, \lambda}(x)=r_{n}^{(2)} \circ s_{n}(x) .
$$

Here we would like to verify Theorem 11 again by making use of (66). From (56) and (65), we see that

$$
\begin{aligned}
r_{n}^{(2)}(x) & =\sum_{k=0}^{n} \sum_{l=0}^{n} S_{1, \lambda}(n, l) S_{1, \lambda}(l, k) x^{k} \\
& =\sum_{k=0}^{n} \sum_{l=k}^{n} S_{1, \lambda}(n, l) S_{1, \lambda}(l, k) x^{k} \\
& =\sum_{k=0}^{\infty} S_{J, \lambda}^{(1)}(n, k) x^{k} .
\end{aligned}
$$

Thus, from (65), (66), and (67), we get what we wanted as follows:

$$
G_{n, \lambda}(x)=\sum_{k=0}^{n} S_{J, \lambda}^{(1)}(n, k)(x)_{k, \lambda}
$$

For any positive integer $r$, the degenerate Bernoulli polynomials $\beta_{n,(r)}(x ; \lambda)$ of order $r$ are defined by

$$
\left(\frac{t}{e_{\lambda}(t)-1}\right)^{r} e_{\lambda}^{x}(t)=\sum_{n \geq 0} \beta_{n,(r)}(x ; \lambda) \frac{t^{n}}{n !} .
$$


For $x=0, \beta_{n,(r)}(\lambda)=\beta_{n,(r)}(0 ; \lambda)$ are called the degenerate Bernoulli numbers of order $r$.

In [9], it was shown that

$$
\begin{aligned}
& \sum_{l_{1}, \ldots, l_{m-1}=0}^{n} S_{1, \lambda}\left(n, l_{1}\right) S_{1, \lambda}\left(l_{1}, l_{2}\right) \cdots S_{1, \lambda}\left(l_{m-1}, k\right) \\
& =\sum_{n \geq l_{1} \geq \cdots \geq l_{m-1} \geq k} S_{1, \lambda}\left(n, l_{1}\right) S_{1, \lambda}\left(l_{1}, l_{2}\right) \cdots S_{1, \lambda}\left(l_{m-1}, k\right) \\
& =\sum_{k_{1}+\cdots+k_{m}=n-k}\left(\begin{array}{c}
n-1 \\
k_{1}, \ldots, k_{m}, k-1
\end{array}\right) \prod_{j=1}^{m} \beta_{k_{j},\left(n-\sum_{i=j+1}^{m} k_{i}\right)}(\lambda) .
\end{aligned}
$$

Hence, in the special cases of $m=1$ and $m=2$, we respectively have

$$
\begin{aligned}
& S_{1, \lambda}(n, k)=\left(\begin{array}{l}
n-1 \\
k-1
\end{array}\right) \beta_{n-k,(n)}(\lambda), \\
& S_{J, \lambda}^{(1)}(n, k)=\sum_{k_{1}+k_{2}=n-k}\left(\begin{array}{c}
n-1 \\
k_{1}, k_{2}, k-1
\end{array}\right) \beta_{k_{2},(n)}(\lambda) \beta_{k_{1},\left(n-k_{2}\right)}(\lambda) .
\end{aligned}
$$

\section{Conclusion}

In this paper, we introduced Jindalrae-Stirling numbers of the first and second kinds as extensions of the notions of the degenerate Stirling numbers of the first and second kinds $($ see $[5,10,18,21])$ and found some relations connecting those special numbers, the degenerate Stirling numbers of the first and second kinds and the degenerate Bell numbers and polynomials (see [12, 13, 15, 19, 21, 27]). Then we introduced Jindalrae numbers and polynomials, as an extension of the notion of the degenerate Bell numbers and polynomials, and Gaenari numbers and polynomials and obtained some explicit expressions and identities involving those numbers and polynomials, Jindalrae-Stirling numbers of the first and second kinds, the degenerate Stirling numbers of the first and second kinds, and the degenerate Bell polynomials. In addition, we interpreted our results by means of umbral calculus (see $[6,9,13,29])$.

As to possible applications our results, we would like to mention three things. The first one is their applications to differential equations. In [8], new combinatorial identities for some degenerate special polynomials were found from certain infinite families of linear and nonlinear ordinary differential equations, satisfied by the generating functions of those polynomials. The second one is their applications to probability theory. In $[16,18]$, new identities connecting some special numbers and moments of random variables were derived by using the generating functions of the moments of certain random variables. The last one is their applications to identities of symmetry. In [11], abundant identities of symmetry were derived for various degenerate versions of many special polynomials by using $p$-adic integrals.

It is one of our future projects to continue this line of research, namely study of degenerate versions of some special polynomials and numbers, and to find some of their possible applications to mathematics, science, and engineering. 
Funding

Not applicable.

\section{Availability of data and materials}

Not applicable.

\section{Competing interests}

The authors declare no conflict of interests.

\section{Authors' contributions}

DSK conceived of the framework and structured the whole paper; DSK and TK wrote the paper; LCJ and HL checked the results of the paper and typed the paper; DSK and TK completed the revision of the article. All authors have read and agreed to the published version of the manuscript.

\section{Author details}

'School of Science, Xi'an Technological University, Xi'an, China. ${ }^{2}$ Department of Mathematics, Kwangwoon University, Seoul, Republic of Korea. ${ }^{3}$ Department of Mathematics, Sogang University, Seoul, Republic of Korea. ${ }^{4}$ Graduate School of Education, Konkuk University, Seoul, Republic of Korea.

\section{Publisher's Note}

Springer Nature remains neutral with regard to jurisdictional claims in published maps and institutional affiliations.

Received: 18 April 2020 Accepted: 17 May 2020 Published online: 27 May 2020

\section{References}

1. Araci, S., Acikgoz, M.: A note on the Frobenius-Euler numbers and polynomials associated with Bernstein polynomials. Adv. Stud. Contemp. Math. (Kyungshang) 22(3), 399-406 (2012)

2. Carlitz, L.: A degenerate Staudt-Clausen theorem. Arch. Math. (Basel) 7, 28-33 (1956)

3. Carlitz, L.: Degenerate Stirling, Bernoulli and Eulerian numbers. Util. Math. 15, 51-88 (1979)

4. Choi, S., Kim, B.-M., Kwon, J.: The deformed degenerate q-Euler polynomials and numbers. Ars Comb. 143, 309-319 (2019)

5. Dolgy, D.V., Kim, T.: Some explicit formulas of degenerate Stirling numbers associated with the degenerate special numbers and polynomials. Proc. Jangjeon Math. Soc. 21(2), 309-317 (2018)

6. Jeong, J., Rim, S.H., Kim, B.M.: On finite-times degenerate Cauchy numbers and polynomials. Adv. Differ. Equ. 2015, Article ID 321 (2015)

7. Khan, W.A.: A new class of degenerate Frobenius-Euler-Hermite polynomials. Adv. Stud. Contemp. Math. (Kyungshang) 28(4), 567-576 (2018)

8. Kim, D.S., Kim, T.: Some identities for Bernoulli numbers of the second kind arising from a non-linear differential equation. Bull. Korean Math. Soc. 52(6), 2001-2010 (2015)

9. Kim, D.S., Kim, T., Kwon, H.I., Mansour, T.: Powers under umbral composition and degeneration for Sheffer sequences. Adv. Differ. Equ. 2016, Article ID 66 (2016)

10. Kim, T.: A note on degenerate Stirling polynomials of the second kind. Proc. Jangjeon Math. Soc. 20(3), 319-331 (2017)

11. Kim, T., Kim, D.S.: Identities of symmetry for degenerate Euler polynomials and alternating generalized falling factorial sums. Iran. J. Sci. Technol. Trans. A, Sci. 41(4), 939-949 (2017)

12. Kim, T., Kim, D.S.: Degenerate polyexponential functions and degenerate Bell polynomials. J. Math. Anal. Appl. 487(2), Article ID 124017 (2020)

13. Kim, T., Kim, D.S.: Some identities of extended degenerate $r$-central Bell polynomials arising from umbral calculus. Rev. R. Acad. Cienc. Exactas Fís. Nat., Ser. A Mat. 114(1), Article ID 1 (2020)

14. Kim, D. S., Kim, T.: A note on a new type of degenerate Bernoulli numbers. Russ. Math. Phys. 27(2), 227-235 (2020)

15. Kim, T., Kim, D.S., Dolgy, D.V.: On partially degenerate Bell numbers and polynomials. Proc. Jangjeon Math. Soc. 20, 337-345 (2017)

16. Kim, T., Kim, D.S., Jang, L.-C., Kim, H.Y.: A note on discrete degenerate random variables. Proc. Jangjeon Math. Soc. 23(1), 125-135 (2020)

17. Kim, T., Kim, D.S., Kim, H.Y., Jang, L.-C.: Degenerate poly-Bernoulli numbers and polynomials. Informatica 31(3), 2-8 (2020)

18. Kim, T., Kim, D.S., Kim, H.Y., Kwon, J.: Degenerate Stirling polynomials of the second kind and some applications Symmetry 11(8), Article ID 1046 (2019)

19. Kim, T., Kim, D.S., Kim, H.Y., Kwon, J.: Some identities of degenerate Bell polynomials. Mathematics 8, Article ID 40 (2020)

20. Kim, T., Kim, D.S., Lee, H., Kwon, J.: Degenerate binomial coefficients and degenerate hypergeometric functions. Adv. Differ. Equ. 2020, Article ID 115 (2020)

21. Kim, T., Yao, Y., Kim, D.S., Jang, G.-W.: Degenerate $r$-Stirling numbers and $r$-Bell polynomials. Russ. J. Math. Phys. 25(1), 44-58 (2018)

22. Kruchinin, D.V., Kruchinin, V.V.: Explicit formula for reciprocal generating function and its application. Adv. Stud. Contemp. Math. (Kyungshang) 29(3), 365-372 (2019)

23. Kwon, J., Park, J.-W., Yun, S.J.: A note on degenerate generalized q-Genocchi polynomials. J. Comput. Anal. Appl. 22(5), 923-928 (2017)

24. Lee, J.G., Kim, W.J., Jang, L.-C., Kim, B.M.: A note on modified degenerate $q$-Daehee polynomials and numbers. J. Inequal. Appl. 2019, Article ID 24 (2019)

25. Lim, D., Kwon, J.: A note on poly-Daehee numbers and polynomials. Proc. Jangjeon Math. Soc. 19(2), 219-224 (2016) 
26. Luo, Y.-N., Wuyungaowa: Some combinatorial identities about Daehee sequences. J. Comb. Math. Comb. Comput. $108,75-87$ (2019)

27. Ma, M., Lim, D.: Some identities on the fully degenerate Bell polynomials of the second kind. Adv. Stud. Contemp. Math. (Kyungshang) 30(1), 145-154 (2020)

28. Pyo, S.-S.: Degenerate Cauchy numbers and polynomials of the fourth kind. Adv. Stud. Contemp. Math. (Kyungshang) 28(1), 127-138 (2018)

29. Roman, S.: The Umbral Calculus. Pure and Applied Mathematics, vol. 111. Academic Press, New York (1984)

30. Saif, M., Nadeem, R.: Evaluation of Apostol-Euler based poly Daehee polynomials. Int. J. Appl. Comput. Math. 6(1), Article ID 1 (2020)

31. Srivastava, H.M.: Some families of generating functions associated with the Stirling numbers of the second kind. J. Math. Anal. Appl. 251(2), 752-769 (2000)

Submit your manuscript to a SpringerOpen ${ }^{\odot}$ journal and benefit from:

- Convenient online submission

Rigorous peer review

Open access: articles freely available online

- High visibility within the field

- Retaining the copyright to your article

Submit your next manuscript at $\boldsymbol{\nabla}$ springeropen.com 